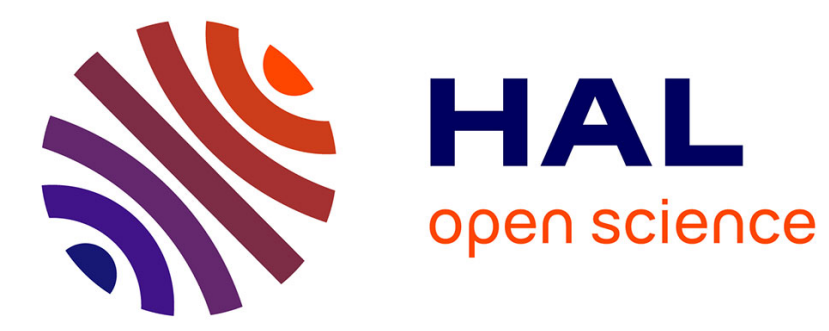

\title{
Dialectes en danger: les derniers locuteurs du mojeño javeriano de Bolivie
}

\author{
F. Rose
}

\section{To cite this version:}

F. Rose. Dialectes en danger: les derniers locuteurs du mojeño javeriano de Bolivie. Faits de langues, 2010, 35-36, pp.255-264. halshs-00724353

\section{HAL Id: halshs-00724353 \\ https://shs.hal.science/halshs-00724353}

Submitted on 4 Sep 2012

HAL is a multi-disciplinary open access archive for the deposit and dissemination of scientific research documents, whether they are published or not. The documents may come from teaching and research institutions in France or abroad, or from public or private research centers.
L'archive ouverte pluridisciplinaire HAL, est destinée au dépôt et à la diffusion de documents scientifiques de niveau recherche, publiés ou non, émanant des établissements d'enseignement et de recherche français ou étrangers, des laboratoires publics ou privés. 


\title{
Dialectes en danger : les derniers locuteurs du mojeño javeriano de Bolivie
}

\author{
Françoise Rose*
}

\section{INTRODUCTION}

Enquêter sur une langue en danger pose inéluctablement la question de la nature des données recueillies et de leur fiabilité : lorsque les locuteurs sont tourmentés par leur sentiment d'insécurité linguistique, le linguiste s'interroge sur la qualité des données. Ajoutez à cela une variation dialectale caractérisée par une grande proximité formelle, mais avec une différence de statut entre dialecte minoré et dialecte dominant, et la question de la fiabilité des données s'élargit avec un risque de confusion entre dialectes.

C'est exactement la situation présentée dans ce chapitre, relatant une courte enquête menée sur un dialecte moribond, le mojeño javeriano, dans le cadre d'une première évaluation de la situation dialectale du mojeño, une langue des basses terres de Bolivie.

Après avoir présenté le mojeño et ses dialectes (Section 1), je présenterai la méthode de linguistique de terrain et mon projet de documentation de la variation dialectale du mojeño (Section 2). Je développerai plus précisément l'enquête réalisée auprès des derniers locuteurs du mojeño javeriano, en expliquant en quoi son déroulement m'était imprévu (Section 3). Dans un dernier temps, j'expliquerai comment une analyse de la situation même de l'enquête a permis d'estimer la fiabilité des données recueillies tout en participant à l'évaluation de la variation dialectale (Section 4).

\section{LE MOJEÑO ET SES DIALECTES}

Le mojeño fait partie de la vingtaine de langues encore parlées dans les basses terres de Bolivie, plus précisément dans le département amazonien du Beni (pour une présentation des langues de la région et de leur documentation/description, cf. le chapitre précédent de Guillaume dans ce volume). Cette langue appartient à la famille arawak, la plus grande famille d'Amérique du Sud, avec quarante langues encore parlées (Aikhenvald 1999). Langue jadis appelée moxo, elle est constituée de quatre variantes: le trinitario, l'ignaciano, le loretano et le javeriano. Le trinitario est parlé dans tout le Territoire Indigène du Parc National

* Laboratoire Dynamique du Langage - CNRS - Université Lumière Lyon 2. 
Isiboro-Sécure (TIPNIS), dans le Territoire Indigène Multi-ethnique (TIM), le long du fleuve Mamoré (dans la ville de Rosario), dans la ville de Trinidad et les villages de San Lorenzo de Mojos, San Francisco de Mojos et leurs environs. L'ignaciano est parlé autour du village de San Ignacio de Mojos, le loretano était parlé à Loreto et le javeriano est le parler de San Javier. Les noms de ces quatre parlers correspondent de toute évidence aux missions construites au XVII ${ }^{\text {ème }}$ siècle par les jésuites (pour une histoire de la région et de ses langues, voir Crevels (2002)). Bien que l'on sache qu'il existait une grande variété linguistique au sein du mojeño à l'arrivée des jésuites, il est difficile d'en faire directement découler la variété dialectale actuelle, d'autant plus que la politique linguistique des missions visait à réduire cette grande diversité à quelques langues générales, dont le Moxo (Saito 2009).

Sur le plan de la vitalité, le trinitario et l'ignaciano sont en danger, le loretano est déjà éteint et le javeriano est moribond, avec une poignée de locuteurs. On comptabilise plus de 20000 mojeños, dont environ seulement 10000 locuteurs de trinitario et d'ignaciano (Crevels 2002). Selon le Recensement Indigène de 1994, parmi les mojeños vivant dans la région amazonienne, 65,3\% étaient monolingues en espagnol, 1,8 \% monolingues dans la langue locale et $32,8 \%$ bilingues. Un autre chiffre révélateur est que $76,6 \%$ de cette population classe l'espagnol comme la langue qu'ils utilisent le plus souvent (Rodríguez Bazán 2000).

La première documentation de la langue mojeño remonte au début du XVIII ${ }^{\text {ème }}$ siècle (Arte de la lengua moxa con su vocabulario y catecismo, du Père Marban (1701), cf Saito (2005)). Plus récemment, des travaux de description linguistique ont vu le jour, mais sur le trinitario et l'ignaciano uniquement. Ces dialectes ont fait l'objet en 1995 de la campagne de normalisation des alphabets sponsorisée par le Sous-secrétariat des Affaires Ethniques, le Ministère de l'Education bolivien et l'UNICEF, dirigée par Colette Grinevald Craig et coordonnée pour ces deux dialectes par Pilar Valenzuela. L'ignaciano est décrit dans une très grosse grammaire (Olza Zubiri et al. 2002), un dictionnaire et des articles de linguistes du SIL (Ott et Ott 1967a, 1967b, 1983). Le trinitario a été décrit dans un manuel et un dictionnaire par un missionnaire évangéliste (Gill, 1957, 1993, [1970]), dans un mémoire de licence d'un étudiant bolivien (Salvatierra 2005) ainsi que dans une grammaire réalisée collectivement par une équipe de professeurs et de locuteurs de la langue, et qui a l'exceptionnelle qualité d'être bilingue espagnol-trinitario (Ibáñez Noza et al . 2007, 2009). Enfin, plusieurs de mes articles sur la morphosyntaxe de la langue vont paraître, notamment Rose (à paraître en 2011, à paraître a, à paraître b).Il existe par ailleurs de nombreux textes publiés par l'équipe pastorale rurale, le Ministère de l'Education, la mission évangélique des Nouvelles Tribus... Le trinitario est enseigné dans certaines écoles primaires, dans un centre social et communautaire, ainsi qu'à l'Université Autonome du Beni à Trinidad et à l'Ecole Normale. Il existe quelques programmes de radio en trinitario et en ignaciano, et un cours de trinitario sur une chaîne de télévision locale. Mes propres travaux sur la langue sontévoqués dans la section suivante. L'unique ouvrage diachronique et dialectologique sur le mojeño est le Diccionario del idioma moxeño a través del tiempo (Becerra 
Casanovas 1980), qui offre des données du mojeño du $18^{\text {ème }}$ siècle et des variétés modernes, mais sans réellement les comparer. La section suivante présente mon projet d'évaluation de la variété dialectale du mojeño.

\section{VERS UNE DOCUMENTATION DE LA VARIATION DIALECTALE}

L'étude des langues à tradition orale, et a fortiori de langues à danger, ne peut se développer sans un effort constant d'amélioration de la documentation de ces langues. Il s'agit d'abord, dans le strict intérêt du linguiste, de construire une base de données à partir de laquelle il pourra élaborer, vérifier puis illustrer ses analyses. Il s'agit ensuite, dans un souci éthique, de sauvegarder des documents représentatifs d'une langue en train de disparaître en les archivant et/ou les diffusant, et de partager avec la population concernée les résultats des recherches pouvant être applicables (Himmelmann 1998, Mithun 1991). La collecte de données sur le terrain répond à une méthodologie particulière. Elle se fait généralement par enregistrement de textes oraux spontanés (narration, exposés, chansons, conversations, blagues...), mais aussi de listes de mots, de paradigmes morphologiques et/ou d'énoncés isolés. Si le travail de transcription, traduction et de sollicitation de données supplémentaires est généralement effectué avec l'aide de quelques informateurs privilégiés, la récolte de données se réalise auprès d'un nombre de locuteurs plus large, prenant en compte les différences de sexe, d'âge et de lieu d'habitation ou de naissance.

Ainsi, j'ai réalisé trois séjours sur le terrain en Bolivie, à Trinidad et à San Lorenzo de Mojos (Septembre-Octobre 2005, Août-Octobre 2006, Avril-Juillet 2008) dans le but de décrire le trinitario avec une perspective typologique et diachronique (une vision générale de mon travail de description de langues amazoniennes est présentée dans une vidéo (Rose 2007)). J'ai recueilli une trentaine de textes au total, des textes de genres différents (contes, récits historiques, conversations, narrations personnelles, exposés, chansons) enregistrés auprès de locuteurs variés (sexe, âge, lieu d'habitation). Ma base de données contient actuellement environ 6 heures d'enregistrement, transcrit, traduit et d'ors et déjà segmenté dans le logiciel Toolbox, comptabilisant au total plus de 1000 phrases. J'ai également recueilli une liste de plusieurs centaines de mots (suivant en partie la liste de Kaufman (1985), une liste plus courte auprès de 7 locuteurs différents pour une étude de la variation, une autre liste enregistrée auprès de 6 locuteurs pour une analyse de l'accent, de nombreux paradigmes morphologiques (intéressants sur le plan morphophonologique) et, pour une compréhension approfondie des textes, de nombreuses notes d'enquête sur le vocabulaire et la morphologie. La base de données lexicale contient plus de 1500 entrées.

Après les deux premiers séjours sur le terrain entièrement consacrés à la collecte de données sur le mojeño trinitario, j'ai souhaité lors de mon troisième séjour prendre en compte la diversité du mojeño, en comparant mes données du trinitario à celles de l'ignaciano, du javeriano et du loretano. Trois raisons justifiaient ce projet : 
- Pour définir clairement mon objet d'étude, il importait de définir la relation entre le trinitario et les parlers des autres communautés mojeños. S'agit-il de langues différentes ou de dialectes d'une même langue ? Posons dès maintenant que le résultat de cette première évaluation est que sans aucun doute, les quatre parlers sont des dialectes de la même langue. L'intercompréhension est importante.

- Sachant que ces parlers étaient en danger d'extinction (Crevels 2002), leur documentation avant leur disparition participe à l'effort de sauvegarde (sur ce thème, voir par exemple Krauss (2007)).

- La comparaison du trinitario parlé actuellement aux données du mojeño du XVII ${ }^{\text {ème }}$ siècle a dévoilé une importante évolution. L'étude des autres parlers dérivés de ce même mojeño fournira probablement des points de comparaison profitables à une étude diachronique.

Ma collecte de données s'était jusque là concentrée sur le trinitario. J'avais eu accès à l'ignaciano uniquement par la lecture des descriptions linguistiques et au javeriano et au loretano uniquement par un dictionnaire comparatif (Becerra Casanovas 1980). A première vue, une comparaison superficielle des quatre dialectes montrait déjà une grande similarité sur le plan lexical, une fois prises en compte les correspondances phonologiques régulières. Sur le plan de la morphosyntaxe, mes premières analyses du trinitario étaient proches du contenu de la grammaire ignaciano (Olza Zubiri et al. 2002). Pour affiner cette comparaison, mon projet était d'enregistrer une même liste de vocabulaire de base en trinitario, ignaciano, javeriano et loretano. L'objectif était, dans un premier temps, d'enregistrer la liste de 150 mots enregistrée auprès de trinitarios par Pilar Valenzuela lors de l'atelier de révision des alphabets, mais cette fois auprès de locuteurs de l'ignaciano, du javeriano et du loretano.

Par ailleurs, quelques remarques métalinguistiques faites par des locuteurs de trinitario laissaient entendre qu'au sein même du trinitario, il y avait des variations suffisamment importantes pour reconnaître le lieu d'origine des locuteurs. La variété parlée par les trinitarios vivant dans le Territoire Indigène du Parc National Isiboro-Secure est considérée comme le "véritable" trinitario, ce que les trinitarios de Trinidad et de San Lorenzo de Mojos expliquent par un mode de vie plus traditionnel, un moindre degré de contact avec l'espagnol, et le fait que les enfants de cette zone pratiquent toujours la langue indigène. Ces remarques m'ont aussi poussée à prévoir une collecte de données dans cette zonelà. Etant donné que la différence avec la variété que j'étudiais semblait a priori minime et n'était pas précisément définie comme relevant du niveau phonétique, phonologique, lexical ou morphosyntaxique, je programmais d'enregistrer dans un premier temps la liste de mots mentionnée ci-dessus. Si la variation était assez minime pour que cette variété participe de manière cohérente à mon objet d'étude (la description du dialecte trinitario), j'envisageais d'enregistrer ensuite du discours spontané.

La réalisation de ces projets s'est révélée, comme souvent sur le terrain, modifiée par les circonstances matérielles et le hasard des rencontres. Ne disposant pas du temps nécessaire à un déplacement en barque jusqu'aux villages trinitarios du Parc National Isiboro-Secure, j'effectuais des enregistrements à 
Trinidad même, auprès de locuteurs originaires de la zone en question, et représentant leurs communautés d'origine dans l'antenne locale d'une institution indigène régionale, la Central de los Pueblos Indigenas del Beni (CPIB). J'ai enregistré une liste de mots et des textes auprès de Placida Muiva Tamo, la présidente de la Sub-central et d'un de ses collègues, Osvaldo Noe Teko. Je n'ai pas noté de différences frappantes avec la variété étudiée jusque là.

Concernant l'ignaciano, j'ai rencontré des locuteurs lors de ma participation comme enseignante à un atelier de formation en linguistique pour instituteurs bilingues des basses terres organisé par l'Université Gabriel René Moreno de Santa Cruz. Les instituteurs suivaient des cours théoriques et pratiques et devaient en parallèle effectuer des recherches sur leur propre langue. La première étape de cette recherche était l'enregistrement de la liste de mots dite de Swadesh. L'un des participants à ce programme, Mariano Matenez Maija, m'a gentiment permis de copier l'enregistrement qu'il avait réalisé auprès de sa mère Matilde Maija Jimenez. Par ailleurs, les discussions entre trinitario et ignaciano lors de cet atelier m'ont permis de confirmer les correspondances régulières, mais importantes auditivement, entre les deux dialectes. Par exemple, aux $/ \mathrm{o} / \mathrm{du}$ trinitario, une voyelle importante du fait de sa présence prépondérante dans la morphologie verbale, correspondent toujours des /a/ en ignaciano, ce qui fait dire aux trinitarios que les ignacianos ne parlent "qu'avec des a". On notera entre autres aussi l'absence en ignaciano de voyelles longues et du phénomène de chute de voyelles.

Concernant le loretano, un déplacement à Loreto, au cœur de la zone loretophone, m'a laissé entendre qu'il n'existait plus aucun locuteur du loretano. Quelques personnes se souviennent de rares mots, mais vraisemblablement j'arrivais trop tard dans le processus de déclin du parler pour mener à bien une enquête. Cependant, ce parler ne restera pas sans documentation. Grâce à un enregistrement réalisé les 5 et 6 octobre 1987 par la sociologue Zulema Lehm et conservé depuis au Centro de Investigación y Documentación para el Desarrollo del Beni (CIDDEBENI), il sera possible de connaître la place de ce parler au sein du mojeño. Zulema Lehm, lors d'une enquête auprès des loretanos, avait interviewé une des dernières locutrices, Manuela Cayaba, alors âgée de plus de 80 ans et décédée depuis. Celle-ci s'exprimait en espagnol lorsque l'enquêtrice l'interrogeait dans cette langue, et en loretano, lorsque le rôle de l'enquêteur était joué par Lorenzo Vare Chavez, un trinitario. Ce dernier s'adressait à Manuela Cayaba en trinitario, et celle-ci répondait en loretano, ce qui démontre à merveille qu'il y a intercompréhension entre les deux dialectes. A première écoute, aucune différence fondamentale n'est perceptible entre les deux variantes. Reste à étudier en détail le discours de Manuela Cayaba. Cet épisode de mon enquête illustre parfaitement l'importance d'effectuer des enregistrements en prenant en compte leur intérêt potentiel pour des chercheurs d'autres disciplines et les futures générations. Zulema Lehm aurait parfaitement pu ne pas enregistrer cette locutrice bilingue en loretano, effacer cette partie de l'enregistrement, ne pas l'archiver dans un lieu sûr, oublier que cet enregistrement existait, ou encore ne pas coopérer avec moi en fouillant l'archive audio pour me prêter la cassette. Cette coopération interdisciplinaire est d'une importance inestimable pour moi, 
qui n'avait aucune possibilité d'accès à ce parler déjà éteint, et je l'espère pour elle, qui verra dans le résultat de mes analyses toute l'importance d'avoir effectué ce travail de documentation et de sauvegarde d'un parler alors moribond.

Enfin, la collecte de données du javeriano a constitué l'épisode le plus surprenant et le plus formateur méthodologiquement pour un chercheur pourtant aguerri à la collecte de données de langues en danger dans des situations d'enquête de terrain parfois complexes.

\section{ENTRETIEN AVEC LES DERNIERS LOCUTEURS DU JAVERIANO : DE SURPRISE EN SURPRISE}

Cette section conte la préparation de l'enquête et sa réalisation, sur un mode volontairement subjectif. Une analyse plus distanciée de la situation d'enquête a eu lieu ultérieurement et sera proposée dans la Section 4 (pour les différentes phases du travail de terrain, Cf. Mondada 1998).

Mon enquête sur le javeriano a été facilitée une fois de plus par l'aide de la sociologue Zulema Lehm, qui m'a procuré le nom d'un habitant de San Javier, M C. En l'absence du président de la Sub-central de San Javier, c'était mon seul contact sur place. Il fut facile à localiser, et par chance, s'est présenté comme un des cinq derniers locuteurs du javeriano. Il fut d'un abord plus que jovial et coopératif, m'adressant la parole dans sa langue dès qu'il sût le but de ma visite et acceptant sans hésitation de m'aider dans ma tâche. Il était d'accord pour me fournir des traductions de mots et enregistrer des textes, proposant même de convoquer d'autres locuteurs. Malheureusement, il s'avéra rapidement que la chaleur de l'accueil était due à un état d'ivresse ne permettant pas d'effectuer une séance de travail dans des conditions saines. Il fut convenu que je le contacterai ultérieurement. J'avais déjà eu le temps de noter quelques correspondances régulières entre le javeriano et le trinitario qui ne gênaient pas l'intercompréhension (même pour la trinitariophone imparfaite que je suis).

Passons outre les difficultés matérielles, un rendez-vous fut pris par téléphone, M C. paraissant toujours aussi coopératif et déterminé à organiser des séances de travail en compagnie d'autres locuteurs. J'appris par ailleurs qu'il enseignait le 'dialecte' à l'école de San Javier. Lorsque j'arrivai au village le jour convenu, j'avais deux heures pour procéder à une petite enquête qui me servirait à proposer une première évaluation de la variation dialectale. Malgré l'accueil chaleureux de l'informateur, je remarquai son attitude fuyante. Il opta pour l'enregistrement de la liste de mots uniquement, en excluant textes ou conversations, préférait que je travaille avec d'autres locuteurs, devait s'occuper des cochons et des poules, etc. Ce furent les premiers signes d'une insécurité linguistique que je commençai alors à démasquer. Puis débuta véritablement la séance de travail, enregistrée dans son intégralité, où M C. (âgé de 54 ans) était d'abord aidé seulement par un ancien (M X., qui avait comme handicap sa surdité et les séquelles d'une fête de village très arrosée), puis par sa mère, Mme G. (âgée de 72 ans). Dès les premiers mots de la liste de vocabulaire de base, je m'aperçus de l'absence de pratique de la langue : malgré tous leurs efforts, les informateurs ne retrouvèrent jamais certains mots et hésitèrent longuement sur d'autres. M C. proposait 
souvent plusieurs formes phonologiquement proches (comme, dans mon intuition, l'aurait fait un étranger ayant jadis appris une langue...). Généralement, il était le premier à proposer une traduction en javeriano du mot recherché, Mme G. confirmant ou corrigeant ensuite sa proposition. Elle-même, bien qu'elle ait oublié de nombreux mots, était tout à fait sûre d'elle lorsqu'un mot lui revenait. En cas de désaccord, lui se ralliait systématiquement à son avis à elle.

Malgré toutes les difficultés de mémoire des interviewés, chaque trouvaille était donc saluée par la satisfaction générale et l'enquête se déroula dans des conditions très agréables, voire ludiques. M X. ne trouva qu'un seul mot, celui pour "tabac", ce qui illustra parfaitement l'intuition que je suivis pendant toute l'enquête, que les mots plus chargés émotionnellement étaient plus faciles à retrouver pour les locuteurs: pas de problème donc pour les insultes et le vocabulaire sexuel, le "menteur" plutôt que la "vérité", et la forme du verbe "boire" qui sortit spontanément fut "je bois beaucoup"! Bien au-delà d'une perte de registres, on peut noter que la rétention du vocabulaire est liée à une forte charge pragmatique.

Après l'enregistrement de la liste de mots, j'avais prévu d'enregistrer un monologue et une conversation. Un des objectifs dans le fait de regrouper plusieurs locuteurs était justement de créer un contexte pour du discours naturel. Mais je me rendis alors compte à quel point le projet de conversation était irréalisable, vu le manque de pratique au quotidien de ces locuteurs. Je demandai cependant à M C. s'il pouvait penser à quelque discours à enregistrer. Après réflexion, sa seule proposition était d'enregistrer l'hymne du village, une chanson en javeriano, ce que nous fîmes. C'est ainsi que se conclut cette séance de travail mouvementée, pleine de surprises, mais finalement satisfaisante pour tous. Les locuteurs étaient contents d'avoir mené à bien cette tâche difficile, les "spectateurs" non locuteurs avaient satisfait leur curiosité de cette situation improbable où une étrangère voulait connaître la langue locale qu'eux ignoraient, et moi d'avoir non seulement recueilli des données sur un parler en danger, de pouvoir donc étudier ce qui s'avérait n'être qu'une distinction dialectale entre le trinitario et le javeriano, mais surtout d'avoir été plongée dans une situation d'enquête terriblement enrichissante.

\section{FIABILITE DES DONNEES : RETOUR ANALYTIQUE SUR LA SITUATION D'ENQUETE}

Immédiatement après l'enquête, je réalisai à quel point cette courte interview se distinguait de toutes mes expériences antérieures. La tâche avait en fait été relativement aisée et agréable, en comparaison par exemple à certaines données d'autres langues que j'avais parfois eu beaucoup de difficultés (matérielles et culturelles) à solliciter. Cependant, le résultat qualitatif et quantitatif de cette collecte me parut bien fragile. Je me rendis compte que même si je savais pertinemment que ce parler n'était plus connu que de quelques personnes, je fus surprise dans l'évaluation des compétences des locuteurs. Je ne m'étais pas préparé mentalement à collaborer avec des personnes ne maîtrisant pas la langue. Je pensais sincèrement pouvoir enregistrer la plupart des 150 mots de la liste et au moins une petite conversation. C'est d'ailleurs à cela que m'avait préparée 
l'attitude de M C., en acceptant d'entrée de jeu de collaborer avec moi. Le fait qu'à notre première rencontre il m'ait adressé la parole dans sa langue, et qu'il ait enseigné la langue à l'école du village me laissait supposer que son usage de la langue était quotidien, qu'il était un idiomista comme on désigne localement les personnes parlant bien leur langue. En réalité, il est fort probable que cette personne n'ait jamais complètement acquis le javeriano ni ne l'ait utilisé comme principal outil de communication (Je reviendrai plus bas sur la classification des trois informateurs dans la typologie des locuteurs). Ce premier bilan suite à l'entretien a donc mis en évidence l'importance de l'attitude, que ce soit celle des locuteurs ou celle des 'étrangers', dans l'image que l'on construit de la langue. Si cet entretien fut plein de surprises pour moi, c'est que cette image d'une langue parlée par peu de locuteurs, mais encore bien vivante pour eux s'est peu à peu déconstruite, à mesure que l'assurance apparente du locuteur cédait place à sa réelle insécurité linguistique.

Après avoir révisé mon analyse de l'usage véritable du javeriano, il fallait aussi réfléchir à l'impact de cette situation sociolinguistique de langue moribonde sur la qualité des données recueillies. Les cours de description des langues à tradition orale et de sociolinguistique que j'avais suivis à l'Université Lumière Lyon2 m'avaient sensibilisée à l'importance de la prise en compte de la situation sociolinguistique. Après avoir revécu l'entretien pendant sa transcription minutieuse, je notai nombre de problèmes influant sur la fiabilité des données.

$\mathrm{Au}$ premier plan, les problèmes de "mémoire" liés à la perte d'usage de la langue ont compromis la collecte de données, tout d'abord au niveau quantitatif, le plus évident. La perte lexicale s'est manifestée de manière indéniable par des blancs, des affirmations du type "j'ai oublié", de longues hésitations ou des autocorrections. Cette perte lexicale en cours a également un impact sur la qualité des données. Par exemple, certaines formes proposées par les informateurs n'ont en fin de compte pas été validées avec certitude et restent donc sujet à caution. Le désaccord fréquent des deux informateurs actifs lors de l'entretien laisse à penser que la même enquête avec un seul locuteur aurait fourni des résultats bien moins nuancés, mais encore plus douteux.

D'autres lacunes qui a priori auraient pu être rattachées à la perte d'usage de la langue pointent en réalité la possibilité que l'informateur principal, M C., n'ait jamais été un locuteur actif de la langue. Ces faits sont moins flagrants. On aura remarqué que le seul texte qu'il pouvait fournir était l'hymne du village. Il s'agit d'un type de connaissance apprise par cœur, chargée identitairement, et qui peut classiquement subsister dans les mémoires des non-locuteurs après la perte totale d'usage de la langue. C'est ce que Sasse (1992) appelle des "résidus", notamment fréquents dans des objectifs spécifiques comme les rituels. Pensons à "et la bobinette cherra" que presque tout français connaît sans jamais l'avoir utilisé dans la vie quotidienne. Un autre point plus gênant pour l'analyse, d'autant plus qu'il n'aurait pas été flagrant pour un enquêteur ne connaissant pas déjà la langue, est que M C. faisait beaucoup d'erreurs de choix morphologique dans les formes fléchies (verbes conjugués et noms possédés). Cette perte morphologique (ou acquisition incomplète de la morphologie) doit être distinguée des erreurs typiques des séances d'enquête du type où le linguiste demande comment on dit 
"je m'assois" et le locuteur répond "assieds-toi". Ici, les formes échangées étaient surprenantes, ce qui donnait l'impression d'un locuteur essayant tant bien que mal de se souvenir d'une forme apprise à l'école ou de manière détachée d'un contexte de communication et essayant tant bien que mal de se souvenir d'une forme. Ici on remarquera encore l'évolution de ma vision de cet informateur dans la typologie des locuteurs (nous suivons ici la typologie aménagée par Bert pour le francoprovençal et l'occitan dans la région du Pilat (Bert 2003) : ce locuteur est passé de la catégorie "locuteur traditionnel", pratiquant sa langue au quotidien et au surcroît érudit capable d'enseigner sa langue, à la catégorie d'un "semilocuteur" (Dorian 1977), n'ayant jamais pratiqué la langue mais l'ayant apprise comme une langue seconde. La tâche de jugement de fiabilité des données est donc complexe, non seulement du fait que les deux informateurs actifs ne sont pas des locuteurs traditionnels, mais en outre au regard de la présence de deux types de locuteurs différents. Si aucun n'est exposé de nos jours à la langue, M C. n'a connu qu'une acquisition partielle de la langue, jusqu'à obtenir des compétences limitées : il s'agit d'un semi-locuteur. Mme G., en revanche est une "ancienne locutrice" : elle a été exposée à la langue essentiellement dans son enfance, jusqu'à un niveau de compétences quasi complet mais n'a eu par la suite qu'une exposition faible à la langue avec un usage épisodique. M X. est difficile à classer dans cette typologie des locuteurs dans l'état actuel de nos connaissances. Concrètement, il ne prononça qu'un seul mot javeriano en deux heures. Son comportement en quasi muet divergeait diamétralement avec la caractérisation comme "locuteur" qu'en avaient fait les autres personnes. Cette classification des derniers locuteurs du javeriano et de leurs usages de la langue illustre de manière cruelle le passage de l'étape "language decay" à l'étape "mort de la langue" dans le modèle en trois phases de mort des langues de Sasse (1992). Lors de cette situation d'enquête sur une langue moribonde, diverses facettes de la perte d'une langue e sont apparues au fur et à mesure de l'enquête: la perte de contexte naturel pour l'apprentissage ou pour l'observation participative, la perte du sens de la norme, la perte du choix des locuteurs, la perte de confiance de certains types de locuteurs, etc (Grinevald 2006).

Je repensai aussi au fait que M C. avait enseigné la langue à l'école du village. Il n'existe aucune littérature sur le javeriano, ni en javeriano. Mais il est fort probable que le professeur ait utilisé des manuels existant en trinitario, ce qui jette beaucoup de doutes sur toutes les données fournies dont la forme était identique à celle du trinitario. L'hypothèse selon laquelle cet informateur allait parfois puiser dans ses connaissances du trinitario serait corroborée par sa manière à lui de chercher les mots (comme un élève en cours de langue plutôt que comme quelqu'un ayant jadis pratiqué la langue), par les quelques interventions des "spectateurs" de la séance, qui proposaient toujours des formes identiques à celle du trinitario, et par le fait que, dans les cas de désaccord entre lui et Mme G., quand lui proposait une forme semblable au trinitario et elle une forme légèrement différente, c'est finalement la forme qu'elle avait proposée qui fut acceptée par les deux locuteurs comme la forme correcte que je devais noter. La probabilité est donc haute que des mots proposés par M C. soient en fait des mots de trinitario. Il est clair que sociolinguistiquement, le trinitario est le 
dialecte dominant du mojeño, par le nombre de ses locuteurs, par l'inclusion dans la zone trinitariophone de Trinidad, la capitale du département du Beni, par la présence de professeurs de trinitario à l'Université... Ici intervient la question de l'identité. Quelle est la force de l'identité dialectale dans le cas d'un dialecte moribond voisin d'un dialecte bien plus vivace et reconnu ? Il faut de plus prendre en compte l'attitude générale de la population vis-à-vis des langues indigènes : on parle du dialecto sans distinction ni de langues différentes, ni de dialectes à l'intérieur de ces langues. Le risque de ce type d'enquête (encore plus lorsque réalisée par un enquêteur étranger) est celui du nivellement de la variation dialectale, ou de la pure substitution du dialecte en déclin par le dialecte dominant. On aura remarqué que le seul texte que l'informateur s'est enhardi à enregistrer est d'ordre identitaire, marquant même l'identité hyper locale, dialectale. A cela s'ajoute le fait que les locuteurs avaient pu apprécier mon niveau de connaissance du trinitario, et que confronté à ses propres lacunes, le professeur avait la possibilité de me satisfaire en fournissant une forme trinitario, ce que sa mère n'a jamais fait. Le "mélange des dialectes" constitue donc une difficulté supplémentaire dans le jugement de fiabilité des données.

\section{CONCLUSION OU LA MORALE DE L'HISTOIRE...}

Ce chapitre a retracé les difficultés concrètes encourues par une linguiste lors d'une première tentative d'évaluer la variation des parlers d'une région, constituant ainsi une illustration concrète de l'affirmation selon laquelle "the bewilderment of the linguist trained in a tradition that only considers the description of vital languages with healthy native speakers is great." (Grinevald, 1998 : 155). Il a relaté en particulier un court entretien avec les derniers locuteurs d'un dialecte, réalisé sur fond d'une situation sociolinguistique de parlers sur le point de s'éteindre, de dialectes formellement très proches mais avec un dialecte dominant et un autre minoré, avec trois locuteurs non traditionnels face à une enquêtrice étrangère avec un bon niveau de connaissance du dialecte dominant, sans oublier les habituelles difficultés matérielles et relationnelles liées à toute enquête sur une langue à tradition orale. Le résultat était au premier abord décevant quantitativement et douteux qualitativement. Une analyse ultérieure a permis de mettre en évidence de nombreux décalages : pour un des informateurs, décalage de comportement entre son assurance métalinguistique et son insécurité linguistique ou entre ses compétences déclarées et ses compétences réelles; pour sa mère, entre ses compétences et son usage de la langue ; pour le troisième informateur entre l'image qu'en ont ses amis et l'image qu'il m'a donné ... Cette foule de renseignements intéressants sur les derniers locuteurs du parler mojeño javeriano permet d'un côté d'affiner la description de sa situation sociolinguistique, et de l'autre de réviser la qualité des données afin de pouvoir mieux juger leur fiabilité. Ce court entretien à première vue peu concluant a en fin de compte permis de faire progresser l'évaluation de la variation dialectale du mojeño, dont le trinitario, l'ignaciano, le loretano et le javeriano sont de toute évidence quatre dialectes. 